\title{
FRACTAL DIMENSION FOR FRACTAL STRUCTURES
}

\author{
M.A SÁNCHEZ-GRANERO AND M. FERNÁNDEZ-MARTÍNEZ
}

\begin{abstract}
The main goal of this paper has a double purpose. On the one hand, we propose a new definition in order to compute the fractal dimension of a subset respect to any fractal structure, which completes the theory of classical box-counting dimension. Indeed, if we select the so called natural fractal structure on each euclidean space, then we will get the box-counting dimension as a particular case. Recall that box-counting dimension could be calculated over any euclidean space, although it can be defined over any metrizable one. Nevertheless, the new definition we present can be computed on an easy way over any space admitting a fractal structure. Thus, since a space is metrizable if and only if it supports a starbase fractal structure, our model allows to classify and distinguish a much larger number of topological spaces than the classical definition.
\end{abstract}

On the other hand, our aim consists also of studying some applications of effective calculation of the fractal dimension over a kind of contexts where the box-counting dimension has no sense, like the domain of words, which appears when modeling the streams of information in Kahn's parallel computation model. In this way, we show how to calculate and understand the fractal dimension value obtained for a language generated by means of a regular expression, and also we pay attention to an empirical and novel application of fractal dimension to natural languages.

\section{INTRODUCTION}

Since the concept of fractal was introduced by Benoît Mandelbrot at early seventies, the study and analysis of this kind of non-linear objects has become more and more important. In this way, fractals have been applied to a diverse spectrum of fields in science, such as the diagnosis of diseases (like osteoporosis ([21]) or cancer ([1]), dynamical systems ([24]), ecology ([3]), earthquakes ([15]), detection of eyes in

2010 Mathematics Subject Classification. Primary 28A80; Secondary 68Q55, $54 \mathrm{E} 35$.

Key words and phrases. Fractal, fractal structure, generalized-fractal space, fractal dimension, self- similar set, box-counting dimension, open set condition, domain of words, regular expression.

The first author acknowledges the support of the Spanish Ministry of Science and Innovation, grant MTM2009-12872-C02-01. 
human face images (19]), and the analysis of the human retina ([18]), just to name a few. Furthermore, topology allows the study of fractals from both theoretical and applied points of view, by means of fractal structures, which were first sketched in [2]. In this way, the introduction of fractal structures has allowed to formalize some topics on fractal theory, and its use leads to connect diverse and interesting subjects on general topology like transitive quasi-uniformities, non-archimedean quasimetrization, metrization, topological and fractal dimensions, selfsimilar sets and even space filling curves (see [22]). Indeed, one of the main tools applied to the study of fractals is the fractal dimension, understood as the classical box-counting dimension, since it is a single quantity which offers some information about the complexity of a given set.

One of the purposes of this paper consists of providing a new definition in order to calculate the fractal dimension of a set respect to any fractal structure. With this in mind, we examine some properties of that definition and relate it with the box-counting dimension where the latter can be defined, by providing some conditions over the elements of the fractal structure we select. In this way, we also study the new fractal dimension over self-similar sets, which constitute a kind of fractals which always present a fractal structure in a natural way.

Recall that a fractal structure is a countable collection of coverings which constitutes an approximation of the whole space by a discrete sequence of levels. Thus, it is the perfect place to provide a definition of fractal dimension. The first notion of dimension we propose depends only on the fractal structure we select but not on any metric. Notice that it may seems counterintuitive at a first glance, since spaces as the Cantor set have dimension one with respect to some fractal structure, but this fact is a consequence of depending only on the fractal structure and not on any metric we can consider on the space. We explain this situation in remark 4.6. The second notion of dimension we propose depends not only on the fractal structure but also on a metric or a distance function. In this way, the second version of fractal dimension agrees with the first one when working with the semimetric associated with the fractal structure. Thus, we can use the second notion if we need to consider the size of the elements of each level of the fractal structure.

On the other hand, one of the main advantages of box-counting dimension consists of the easiness of its effective calculation and empirical estimation over the euclidean spaces, though it can also be defined over the metrizable ones. However, this classical definition does not have so good analytical properties as other definitions present, like the Hausdorff dimension. Moreover, our new definitions in order to compute 
the fractal dimension of a subset respect to any fractal structure can be easily calculated over any space admitting a fractal structure. In this way, recall that a topological space is metrizable if and only if it admits a starbase fractal structure ([6, Theorem 4.2]), so that the new definitions allow to classify and distinguish a larger volume of topological spaces than the box-counting model. Note that the box-counting dimension is just a particular case of the new fractal dimension definitions, since it suffices with taking the natural fractal structure on any euclidean space which we formally introduce in section 3 .

The other main goal that this paper provides consists of computing the fractal dimension in interesting contexts where the box-counting dimension has no sense, as the domain of words which appears when modeling the streams of information in Kahn's parallel computation model (see [17] and [20]). Thus, we calculate and explain the fractal dimension of a language generated by means of a regular expression where infinite length words could exist. Moreover, we present another empirical application of fractal dimension to the study of the fractal complexity of any natural language, which allows to compare natural languages, and even to analyze the variety of words used in any text or book written in any language. It is also possible to quantify the complexity of a translation of any text respect to its original version. Finally, we show how fractal dimension is a useful tool in order to study the efficiency of an encoding system as the standard BCD.

\section{Preliminaries}

Let's start with some preliminary topics.

\subsection{Fractal structures, self-similar sets and quasipseudomet-} rics. The main purpose of this section consists of recalling some notations and basic notions that will be useful in this paper.

In this way, the key concept we are going to use is about fractal structures. Nevertheless, although a more natural use of them is in the study of fractals, and in particular self-similar sets (see [9]), its introduction was first motivated in order to characterize non-archimedeanly quasimetrization (see [5]). The use of fractal structures provides a powerful tool in order to study new models for a fractal dimension definition, since they will allow to distinguish and classify a larger volume of spaces than by using the classical definitions of fractal dimension (which will be obtained as a particular case), that only work over metrizable spaces. So that, these kind of topological spaces constitutes a perfect place in order to develop a theory on fractal dimension. 
Let $\Gamma$ be a covering of $X$. Thus, we will denote $S t(x, \Gamma)=\cup\{A \in \Gamma$ : $x \in A\}$ and $U_{x \Gamma}=X \backslash \cup\{A \in \Gamma: x \notin A\}$. Furthermore, if $\boldsymbol{\Gamma}=\left\{\Gamma_{n}\right.$ : $n \in \mathbb{N}\}$ is a countable family of coverings of $X$, then we will denote $U_{x n}=U_{x \Gamma_{n}}, \mathcal{U}_{x}^{\Gamma}=\left\{U_{x n}: n \in \mathbb{N}\right\}$ and $S t(x, \boldsymbol{\Gamma})=\left\{S t\left(x, \Gamma_{n}\right): n \in \mathbb{N}\right\}$.

The next definition was introduced in [5].

Definition 2.1. Let $X$ be a topological space. A pre-fractal structure on $X$ is a countable family of coverings (called levels) $\boldsymbol{\Gamma}=\left\{\Gamma_{n}: n \in \mathbb{N}\right\}$ such that $\mathcal{U}_{x}^{\Gamma}$ is an open neighborhood base of every point $x \in X$.

Moreover, if $\Gamma_{n+1}$ is a refinement of $\Gamma_{n}$ (which can be denoted by $\Gamma_{n+1} \preceq$ $\left.\Gamma_{n}\right)$, such that for all $x \in A$ with $A \in \Gamma_{n}$, there exists $B \in \Gamma_{n+1}$ such that $x \in B \subseteq A$, we will say that $\Gamma$ is a fractal structure on $X$.

If $\boldsymbol{\Gamma}$ is a (pre-) fractal structure on $X$, then we will say that $(X, \boldsymbol{\Gamma})$ is a generalized (pre-) fractal space, or simply a (pre-) GF-space. If there is no doubt about $\boldsymbol{\Gamma}$, then we will say that $X$ is a (pre-) GF-space.

Remark 2.2. Note that the levels we use in order to define a fractal structure $\boldsymbol{\Gamma}$ are not coverings in the usual sense, since we are going to enable the possibility that could exist elements on any level of the fractal structure which can appear two times or more when determining the whole family $\boldsymbol{\Gamma}$. For instance, $\Gamma_{1}=\left\{\left[0, \frac{1}{2}\right],\left[\frac{1}{2}, 1\right],\left[0, \frac{1}{2}\right]\right\}$ could be the first level of a given fractal structure $\boldsymbol{\Gamma}$ defined over the closed unit interval.

Note also that if $\boldsymbol{\Gamma}$ is a pre-fractal structure, then any of its levels is a closure preserving closed covering for each(see [[7],Prop. 2.4]).

If $\boldsymbol{\Gamma}$ is a fractal structure on $X$ and $S t(x, \boldsymbol{\Gamma})$ is a neighborhood base of $x$ for all $x \in X$, we will call $\Gamma$ a starbase fractal structure. In general, if $\Gamma_{n}$ has the property $P$ for all $n \in \mathbb{N}$, and $\Gamma=\left\{\Gamma_{n}: n \in \mathbb{N}\right\}$ is a fractal structure on $X$, we will say that $\Gamma$ is a fractal structure with the property $P$, and that $(X, \boldsymbol{\Gamma})$ is a GF-space with the property $P$. For instance, if $\Gamma_{n}$ is a finite covering for all natural number $n$ and $\Gamma$ is a fractal structure on $X$, then we will say that $\Gamma$ is a finite fractal structure on $X$, and that $(X, \boldsymbol{\Gamma})$ is a finite GF-space.

On the other hand, we also recall the definition of self-similar set provided by Hutchinson (see [16]).

Definition 2.3. Let $I=\{1, \ldots, m\}$ be a finite index set and let $\left\{f_{i}: i \in I\right\}$ be a family of contractive mappings defined from a complete metric space $X$ into itself. Then there exists a unique non-empty compact subset $K$ of $X$ such that $K=\cup_{i \in I} f_{i}(K)$, which is called $a$ self-similar set.

In classical non-linear theory, $\left(X,\left\{f_{i}: i \in I\right\}\right)$ is called an iterated function scheme (which we will denote by IFS for short), and the 
self-similar set $K$, as the atractor of that IFS. Next, we provide an interesting example which describes analytically the so called Sierpinski's gasket, which is a typical example of a strict self-similar set.

Example 1. Let $I=\{1,2,3\}$ be a finite index set and let $\left\{f_{i}: i \in I\right\}$ be a finite set of similarities over the euclidean plane which are defined by

$$
f_{i}(x, y)=\left\{\begin{array}{lll}
\left(\frac{x}{2}, \frac{y}{2}\right) & \text { if } & i=1 \\
f_{1}(x, y)+\left(\frac{1}{2}, 0\right) & \text { if } & i=2 \\
f_{1}(x, y)+\left(\frac{1}{4}, \frac{1}{2}\right) & \text { if } & i=3
\end{array}\right.
$$

for all $(x, y) \in \mathbb{R}^{2}$. Thus, the Sierpinski's gasket is determined on an unique way as the non-empty compact subset verifying the next Hutchinson's equation: $K=\cup_{i \in I} f_{i}(K)$. In this way, note that each component $f_{i}(K)$ is a self-similar copy of the atractor of the IFS $\left(\mathbb{R}^{2},\left\{f_{i}\right.\right.$ : $i \in I\})$.

To consult a procedure in order to approach self-similar sets, see [4] and [10]. Self-similar sets constitute an interesting kind of fractals that are characterized by having a fractal structure in a natural way, which was first sketched in [2]. Indeed, that paper becomes the origin of the term fractal structure. Next, we present the description of such fractal structure (see [9]).

Definition 2.4. Let $I=\{1, \ldots, m\}$ be a finite index set, and let $\left(X,\left\{f_{i}: i \in I\right\}\right)$ be an IFS whose associated self-similar set is $K$. The natural fractal structure on $K$ can be defined as the countable family of coverings $\boldsymbol{\Gamma}=\left\{\Gamma_{n}: n \in \mathbb{N}\right\}$, where $\Gamma_{n}=\left\{f_{\omega}^{n}(K): \omega \in I^{n}\right\}$ for every natural number $n$. Here for all $n \in \mathbb{N}$ and all $\omega=\omega_{1} \omega_{2} \ldots \omega_{n} \in I^{n}$, we denote $f_{\omega}^{n}=f_{\omega_{1}} \circ \ldots \circ f_{\omega_{n}}$.

Remark 2.5. Another available description for this fractal structure is as follows: $\Gamma_{1}=\left\{f_{i}(K): i \in I\right\}$ and $\Gamma_{n+1}=\left\{f_{i}(A): A \in \Gamma_{n}, i \in I\right\}$ for all $n \in \mathbb{N}$.

On example 1 we described analytically the IFS whose associated self-similar set is the Sierpinski's triangle. Next, we are going to present the natural fractal structure associated with this strict self-similar set.

Example 2. The natural fractal structure associated with the Sierpinski's triangle can be described as the countable family of coverings $\boldsymbol{\Gamma}=\left\{\Gamma_{n}: n \in \mathbb{N}\right\}$, where $\Gamma_{1}$ is the union of three equilateral "triangles" with sides equal to $\frac{1}{2}, \Gamma_{2}$ consists of the union of $3^{2}$ equilateral "triangles" with sides equal to $\frac{1}{2^{2}}$, and in general, $\Gamma_{n}$ is the union of $3^{n}$ equilateral "triangles" whose sides are equal to $\frac{1}{2^{n}}$. Furthermore, this is a finite starbase fractal structure with $\operatorname{Ord}(\boldsymbol{\Gamma})=1$. 
Moreover, recall that a quasipseudometric on a set $X$ is a nonnegative real-valued function $d$ on $X \times X$ such that for all $x, y, z \in X$, verifies the following two conditions: (i) $d(x, x)=0$ and (ii) $d(x, y) \leq$ $d(x, z)+d(z, y)$. If in addition $d$ satisfies also the next one: (iii) $d(x, y)=d(y, x)=0$ iff $x=y$, then $d$ is called a quasi-metric. In particular, a non-archimedean quasipseudometric is a quasipseudometric which also verifies that $d(x, y) \leq \max \{d(x, z), d(z, y)\}$ for all $x, y, z \in X$. Further, we have that each quasipseudometric $d$ on $X$ generates a quasiuniformity $\mathcal{U}_{d}$ on $X$ which has as a base the family of sets of the form $\left\{(x, y) \in X \times X: d(x, y)<2^{-n}\right\}, n \in \mathbb{N}$. Then the topology $\tau\left(\mathcal{U}_{d}\right)$ induced by $\mathcal{U}_{d}$ will be denoted simply by $\tau(d)$. Therefore, a space $(X, \tau)$ is said to be (non-archimedeanly) quasipseudometrizable if there exists a (non-archimedean) quasipseudometric $d$ on $X$ such that $\tau=\tau(d)$. Recall that the theory of quasiuniform spaces is covered in [13.

\subsection{The classical definition of fractal dimension over euclidean}

spaces. Fractal dimension is one of the main tools used in order to study fractals, since it is a single value which provides information about its complexity and the irregularities it presents when being examined with enough level of detail. In this way, fractal dimension is usually understood as the classical box-counting dimension. The latter has been also known as information dimension, Kolmogorov entropy, capacity dimension, entropy dimension, metric dimension, ... etc. Note that the box-counting definition is better from an applied point of view than other theoretical ones, like Hausdorff dimension, since the easiness of its effective calculation an empirical estimation. Nevertheless, box-counting dimension have not so good theoretical properties as Hausdorff dimension, for instance. The basic theory on box-counting dimension can be found in [11. Thus, the (lower/upper) box-counting dimensions of a subset $F \subset \mathbb{R}^{d}$ are given by the following (lower/upper) limit:

$$
\operatorname{dim}_{B}(F)=\lim _{\delta \rightarrow 0} \frac{\log N_{\delta}(F)}{-\log \delta}
$$

where $\delta$ is the scale used in the study of $F$ and $N_{\delta}(F)$ can be calculated on an equivalent way as one of the following quantities (see [11, Equivalent definitions 3.1]):

(1) the number of $\delta$-cubes that meet $F$. Recall that a $\delta$-cube in $\mathbb{R}^{d}$ is a set of the form $\left[k_{1} \delta,\left(k_{1}+1\right) \delta\right] \times \ldots \times\left[k_{d} \delta,\left(k_{d}+1\right) \delta\right]$ where $k_{i}$ are integers for all $i \in\{1, \ldots, d\}$.

(2) the number of $\delta=\frac{1}{2^{n}}-$ cubes that intersect $F$, with $n \in \mathbb{B}$.

(3) the smallest number of sets of diameter at most $\delta$ that cover $F$.

(4) the largest number of disjoint balls of radius $\delta$ centered on $F$. 
Note also that the limit described at 2.2 can be discretized by means of $\delta=\frac{1}{2^{n}}$. In this way, $N_{\delta}(F)$ is just the number of elements of each level $\Gamma_{n}$ of the fractal structure which meet $F$. Moreover, the box-counting dimension can be estimated as the slope of a log-log graph plotted over a suitable discrete collection of scales $\delta$.

Hence a natural idea arises: we can propose a new definition of fractal dimension for any fractal structure which generalizes the classical box-counting dimension and allows to classify and distinguish a larger volume of spaces than by means of the classical box-counting dimension definition. Thus, it also results useful in order to calculate the fractal dimension over another kind of spaces, such as the non-euclidean ones, where the box-counting dimension can have no sense. Thus, we show some interesting applications of this fact on section 5, where the boxcounting dimension cannot been applied in the context of domains of words. Nevertheless, our new definition in order to compute the fractal dimension of a subset allows to calculate and illustrate it.

On the other hand, the next remark results useful in this paper.

Remark 2.6. In order to calculate the (lower/upper) box-counting dimensions of any subset $F$ of a space $X$, it suffices with taking limits as $\delta \rightarrow 0$ by means of a decreasing sequence $\left\{\delta_{n}\right\}_{n \in \mathbb{N}}$ verifying that $\delta_{n+1} \geq c \delta_{n}$ for all $n \in \mathbb{N}$, where $c \in(0,1)$ is a suitable constant.

\section{Generalized Definition of Fractal Dimension for a SUBSET RESPECT TO ANY FRACTAL STRUCTURE}

The starting point of the fractal dimension theory for fractal structures begins by taking into account the euclidean metrizable space $\mathbb{R}^{d}$ as well as the equivalent definition 2 for the quantity $N_{\delta}(F)$ that appears at the box-counting introduction seen in subsection 2.2. Indeed, we can construct a fractal structure $\Gamma$ on any euclidean space $\mathbb{R}^{d}$ in a natural way verifying some desirable properties: it is a locally finite tiling starbase fractal structure which has a finite order. Indeed, it is going to be the so called natural fractal srtucture over any euclidean space, whose definition is as follows.

Definition 3.1. The natural fractal structure on the euclidean space $\mathbb{R}^{d}$ is defined as the countable family of coverings $\Gamma=\left\{\Gamma_{n}: n \in \mathbb{N}\right\}$, whose levels are given by $\Gamma_{n}=\left\{\left[\frac{k_{1}}{2^{n}}, \frac{k_{1}+1}{2^{n}}\right] \times\left[\frac{k_{2}}{2^{n}}, \frac{k_{2}+1}{2^{n}}\right] \times \ldots \times\left[\frac{k_{d}}{2^{n}}, \frac{k_{d}+1}{2^{n}}\right]\right.$ : $\left.k_{i} \in \mathbb{Z}, i \in\{1, \ldots, d\}\right\}$ for all $n \in \mathbb{N}$.

Note that the natural fractal structure $\boldsymbol{\Gamma}$ on the euclidean space $\mathbb{R}^{d}$ is just the tiling consisting of $\frac{1}{2^{n}}$-cubes for all natural number $n$ on $\mathbb{R}^{d}$ which allows to calculate the box-counting dimension of any subset of such space. In this way, we are going to propose a new definition 
of fractal dimension on a generic $\mathrm{GF}$-space $(X, \boldsymbol{\Gamma})$ which generalizes the box-counting one if we use the natural fractal structure on the euclidean space $\mathbb{R}^{d}$. However, as happens with box-counting dimensions, our model for a generic fractal dimension is going to be defined using upper and lower limits. Nevertheless, one of the main advantages it presents, consists of the easiness of its effective calculation and theoretical interpretation. Taking it into account, we can consider a larger volume of fractal structures than box-counting dimension, in order to calculate the fractal dimension of a given set. The key concept of this section is defined below.

Definition 3.2. Let $(X, \Gamma)$ be a $G F$-space and let $N_{n}(F)$ be the number of elements of $\mathcal{A}_{n}(F)$ for all $n \in \mathbb{N}$. Thus, the (lower/ upper) fractal dimensions I of a non-empty bounded subset $F$ of $X$ respectively, are defined as the (lower/upper) limit:

$$
\operatorname{dim}_{\boldsymbol{\Gamma}}^{1}(F)=\lim _{n \rightarrow \infty} \frac{\log N_{n}(F)}{n \log 2}
$$

if this limit exists, where $\mathcal{A}_{n}(F)$ is the next family of elements on each level of the fractal structure $\boldsymbol{\Gamma}$, for all natural number $n$ :

$$
\mathcal{A}_{n}(F)=\left\{A \in \Gamma_{n}: A \cap F \neq \emptyset\right\}
$$

In order to estimate the fractal dimension from a computational point of view, we can calculate the slope of the regression line on a log-log graph, just like with the box-counting dimension estimation. In this way, the next result allows to calculate the box-counting dimension of any subset $F \subset \mathbb{R}^{d}$ by means of the fractal dimension I formula: it suffices with counting the number of $\frac{1}{2^{n}}$-cubes for all $n \in \mathbb{N}$, just like the box counting dimension with equivalent definition (2) for $N_{\delta}(F)$. Thus, the proof of the following result becomes straightforward.

Theorem 3.3. Let $\boldsymbol{\Gamma}$ be the natural fractal structure on the euclidean space $\mathbb{R}^{d}$ and let $F$ be a subset of $\mathbb{R}^{d}$. Then the (lower/upper) boxcounting dimension and the (lower/upper) fractal dimension I of $F$ are equal.

Hausdorff dimension constitutes the reference of any definition of fractal dimension. In this way, we can check some of its analytical properties for the fractal dimension I definition (see [11, Chapter 3]). Indeed, we have the following proposition.

Proposition 3.4. Let $(X, \Gamma)$ be a GF-space. Then,

(1) Both $\underline{\operatorname{dim}}_{\Gamma}^{1}$ and $\overline{\operatorname{dim}}_{\Gamma}^{1}$ are monotonic.

(2) $\overline{\operatorname{dim}}_{\Gamma}^{1}$ is finitely stable.

(3) Neither $\underline{\operatorname{dim}}_{\Gamma}^{1}$ nor $\overline{\operatorname{dim}}_{\Gamma}^{1}$ is countably stable. 
(4) There exists countable sets $F \subset X$ such that $\operatorname{dim}_{\Gamma}^{1}(F) \neq 0$.

(5) There exists a locally finite tiling starbase fractal structure $\Gamma$ with finite order on a suitable space $X$ such that $\operatorname{dim}_{\Gamma}^{1}(F) \neq$ $\operatorname{dim}_{\Gamma}^{1}(\bar{F})$ for a given subset $F \subset X$.

Proof. The more straightforward items are left to the reader.

(4) Recall that the countable stability property for a dimension function $\operatorname{dim}$ means that $\operatorname{dim}\left(\cup_{i \in I} F_{i}\right)=\sup _{i \in I} \operatorname{dim}\left(F_{i}\right)$, where $\left\{F_{i}\right\}_{i \in I}$ is a countable family of subsets of $X$. Therefore, consider $X=[0,1]$ with $F=\mathbb{Q} \cap[0,1]$, and let $\Gamma$ be the natural fractal structure on $\mathbb{R}$ induced in $[0,1]$, which can be described by $\Gamma_{n}=\left\{\left[\frac{k}{2^{n}}, \frac{k+1}{2^{n}}\right]: k \in\left\{0,1, \ldots, 2^{n}-1\right\}\right\}$ for all $n \in \mathbb{N}$. Then, it is clear that $N_{n}(F)=2^{n}$, so that $\operatorname{dim}_{\boldsymbol{\Gamma}}^{1}(F)=1$. Further, note that (4) implies (3).

(5) Indeed, consider the fractal structure $\boldsymbol{\Gamma}=\left\{\Gamma_{n}: n \in \mathbb{N}\right\}$ with $\Gamma_{n}=\left\{\left[\frac{k}{2^{n}}, \frac{k+1}{2^{n}}\right] \times\{0\}: k \in\left\{0,1, \ldots, 2^{n}-1\right\}\right\} \cup\left\{\left\{\frac{1}{2^{m}}\right\} \times\left[\frac{k}{2^{n}}, \frac{k+1}{2^{n}}\right]:\right.$ $\left.k \in\left\{0,1, \ldots, 2^{n}-1\right\}, m \in \mathbb{N}\right\}$ for all natural number $n$, on the space $X=([0,1] \times\{0\}) \cup\left\{\left\{\frac{1}{2^{n}}\right\} \times[0,1]: n \in \mathbb{N}\right\}$. Take also $F=\bigcup_{k \in \mathbb{N}}\left(\frac{1}{2^{k+1}}, \frac{1}{2^{k}}\right) \times\{0\}$ as a subset of $X$. Note that $\operatorname{Ord}(\boldsymbol{\Gamma})=2$. On the other hand, it is clear that $\bar{F}=[0,1] \times\{0\}$, so that $N_{n}(F)=2^{n}$ and $N_{n}(\bar{F})=\infty$ for all natural number $n$, which implies that $\operatorname{dim}_{\Gamma}^{1}(F)=1$ and $\operatorname{dim}_{\Gamma}^{1}(\bar{F})=\infty$.

The next question we are going to investigate consists of the possibility of determining an approximation of the box-counting dimension of a subset on a metric space, in terms of its fractal dimension I. In this way, we define the diameter of each level of a fractal structure $\boldsymbol{\Gamma}$ as well as the diameter of a subset in a given level of $\boldsymbol{\Gamma}$ as follows.

Definition 3.5. Let $\boldsymbol{\Gamma}$ be a fractal structure on a metric space $(X, \rho)$, and let $F$ be a subset of $X$. Then, the diameter of each level $\Gamma_{n}$ of the fractal structure $\boldsymbol{\Gamma}$ is defined as

$$
\delta\left(\Gamma_{n}\right)=\sup \left\{\delta(A): A \in \Gamma_{n}\right\}
$$

and the diameter of $F$ on each level $\Gamma_{n}$ of the fractal structure $\Gamma$ is given as the quantity

$$
\delta\left(F, \Gamma_{n}\right)=\sup \left\{\delta(A): A \in \mathcal{A}_{n}(F)\right\}
$$

Note that starbase fractal structures lead to GF-spaces with some desirable properties. Taking it as well as definition 3.5 into account, we have found a condition over the diameters of each level of the fractal 
structure in order to get this kind of topological spaces. The proof of the next theorem results straightforward.

Proposition 3.6. Let $\boldsymbol{\Gamma}$ be a fractal structure on a compatible metric (or quasimetric) space $(X, \rho)$, and suppose that $\delta\left(\Gamma_{n}\right) \rightarrow 0$. Then $\boldsymbol{\Gamma}$ is starbase.

Proof. Indeed, it suffices with checking that $S t(x, \boldsymbol{\Gamma})$ is a neighborhood base of $x$ for all $x \in X$. First of all, it is clear that $x \in U_{x n} \subset$ $\operatorname{St}\left(x, \Gamma_{n}\right)$ for all $x \in X$ and all natural number $n \in \mathbb{N}$, with $U_{x n}$ being a neighborhood of $x$ about the topology associated with the fractal structure $\Gamma$. On the other hand, let $x \in X$ be a fixed point on $X$, and let $\varepsilon>0$. Since $\delta\left(\Gamma_{n}\right) \rightarrow 0$, then there exists a natural number $n_{0} \in \mathbb{N}$ such that $\delta\left(\Gamma_{n}\right)<\varepsilon$ for all $n \geq n_{0}$. Hence, let $m \in \mathbb{N}$ be a natural number such that $m \geq n_{0}$, and consider $S t\left(x, \Gamma_{m}\right)$. Then, for all $y \in S t\left(x, \Gamma_{m}\right)$ there exists $A \in \Gamma_{m}$ with $x \in A$, such that $y \in A$. Moreover, as $\delta\left(\Gamma_{m}\right)<\varepsilon$, we have that $\rho(x, y)<\varepsilon$, namely, $y \in B_{\rho}(x, \varepsilon)$. Accordingly, there exists $m \in \mathbb{N}$ such that $S t\left(x, \Gamma_{m}\right) \subset B_{\rho}(x, \varepsilon)$.

A very natural condition which a fractal structure could verify, consists of the fact that the sequence of diameters of each level of the fractal structure $\boldsymbol{\Gamma}$ decreases on a geometric way, which constitutes the main idea in the following theorem.

Theorem 3.7. Let $\boldsymbol{\Gamma}$ be a fractal structure on a metric space $(X, \rho)$ with $F$ being a subset of $X$, and suppose that there exists a constant $c \in(0,1)$ such that the next condition is verified:

$$
\delta\left(F, \Gamma_{n+1}\right) \leq c \delta\left(F, \Gamma_{n}\right)
$$

for all natural number $n$. Take the constant $\gamma_{c}=\frac{-\log 2}{\log c}$. Then,

(1) $\overline{\operatorname{dim}}_{B}(F) \leq \gamma_{c} \cdot \overline{\operatorname{dim}}_{\Gamma}^{1}(F)$.

(2) $\underline{\operatorname{dim}}_{B}(F) \leq \gamma_{c} \cdot \underline{\operatorname{dim}}_{\Gamma}^{1}(F)$.

(3) Moreover, if there exist both box-counting dimension and fractal dimension $I$ of $F$, then $\operatorname{dim}_{B}(F) \leq \gamma_{c} \cdot \operatorname{dim}_{\Gamma}^{1}(F)$.

Proof. In order to calculate the box-counting dimension of $F$, let $N_{\delta}(F)$ be as (3) on equivalent box-counting definition seen at preliminary subsection 2.2 ,

(1) Taking the geometric decreasing of the diameters of each level of the fractal structure $\Gamma$ into account, we affirm that there exists a constant $c \in(0,1)$ such that $\delta\left(F, \Gamma_{n}\right) \leq c^{n-1} \cdot \delta\left(F, \Gamma_{1}\right)$ for all $n \in \mathbb{N}$. Denote $\delta_{n}=c^{n-1} \cdot \delta\left(F, \Gamma_{1}\right)$ as the general term of a decreasing sequence which converges to 0 . Thus, $\overline{\operatorname{dim}}_{B}(F)=$ 
$\varlimsup_{n \rightarrow \infty} \frac{\log N_{\delta_{n}}(F)}{-\log \delta_{n}} \leq \varlimsup_{n \rightarrow \infty} \frac{\log N_{n}(F)}{-n \log c}=\gamma_{c} \cdot \varlimsup_{\lim _{n \rightarrow \infty}} \frac{\log N_{n}(F)}{n \log 2}$ where remark 2.6 is used in the first equality.

(2) Consider the previous sequence $\left\{\delta_{n}\right\}_{n \in \mathbb{N}}$. Since $\underline{\lim }_{\delta \rightarrow 0} \frac{\log N_{\delta}(F)}{-\log \delta} \leq$ $\underline{\lim }_{n \rightarrow \infty} \frac{\log N_{\delta_{n}}(F)}{-\log \delta_{n}}$, then it suffices with applying a similar argument to the former.

Since the sequence of diameters $\delta\left(\Gamma_{n}\right)$ always decreases on a geometric way when working with self-similar sets (by its construction), then we can estimate the box-counting dimension of a self-similar set by means of its fractal dimension I, which is easier to calculate, since we are going to select its natural fractal structure. In this way, we present the next result.

Corollary 3.8. Let $\boldsymbol{\Gamma}$ be the natural fractal structure on a self-similar set $K$ provided with the euclidean distance. Then, the inequalities contained on the theorem 3.7 are verified, with $c$ being the maximum of the contraction factors $c_{i}$ associated with the contractions $f_{i}$ on the IFS $\left(X,\left\{f_{i}: i \in I\right\}\right)$ whose associated self-similar set is $K$.

Fractal dimension I depends on the fractal structure $\boldsymbol{\Gamma}$ we utilize on the space. Indeed, we show it in the following remark.

Remark 3.9. Let $X$ be a subspace of an euclidean space. Then, it is possible to obtain different values for its fractal dimension I, depending on the fractal structure $\boldsymbol{\Gamma}$ we select in order to calculate it.

Proof. First of all, let $\Gamma_{1}=\left\{\Gamma_{1, n}: n \in \mathbb{N}\right\}$ be a fractal structure whose levels are given by $\Gamma_{1, n}=\left\{\left[\frac{k}{2^{n}}, \frac{k+1}{2^{n}}\right]: k \in \mathbb{Z}\right\}$ for all $n \in \mathbb{N}$, as the natural fractal structure on the real line induced on the middle third Cantor set $C$. Thus, by means of theorem 3.3, we have that $\operatorname{dim}_{\boldsymbol{\Gamma}_{1}}^{1}(C)=\operatorname{dim}_{B}(C)=\frac{\log 2}{\log 3}$ (see [11, Example 3.3]). On the other hand, let $\Gamma_{2}$ be the natural fractal structure on $C$ as a self-similar set. Then, an easy calculation leads to $\operatorname{dim}_{\boldsymbol{\Gamma}_{2}}^{1}(C)=1$, since on each level of the fractal structure $\boldsymbol{\Gamma}_{2}$, there are $2^{n}$ subintervals of length equal to $\frac{1}{3^{n}}$.

The theoretical justification of this result is based on the idea that the first version of fractal dimension regards all the elements on each level $\Gamma_{n}$ of the fractal structure $\Gamma$ as having the same "size", namely, $\frac{1}{2^{n}}$. 


\section{Fractal Dimension on GF-Spaces: A SeCond Version}

We have just presented a first method in order to calculate the fractal dimension of a subset. In this way, the use of GF-spaces leads to generalize the box-counting dimension, which only works over metrizable spaces (and in particular, over the euclidean ones), and it enables the use of a larger collection of fractal structures in order to calculate the fractal dimension than the box-counting method. As seen on the previous section, the definition 3.2 of fractal dimension I regards each element on the family $\mathcal{A}_{n}(F)$ as having the same "size", equal to $\delta=\frac{1}{2^{n}}$ for all $n \in \mathbb{N}$. So that, the natural fractal structure of $\delta$-cubes which we use on the euclidean spaces in order to determine the box-counting dimension of a given subset, can be extended to other kinds of tilings such as triangulations on the plane.

Recall also that in remark 3.9 we have shown the fact that fractal dimension I depends on the fractal structure we use on each case. Taking it into account, we are going to propose another model in order to calculate the fractal dimension of any subset on a GF-space, but this time, we pretend that our definition can consider the possibility that the elements on each family $\mathcal{A}_{n}(F)$ could present diameters not necessarily equal to $\frac{1}{2^{n}}$.

To this end, we will use the more general concept of distance function (see [25]):

Definition 4.1. A mapping $d: X \times X \rightarrow \mathbb{R}^{+}$defined on a set $X$ is said to be a distance function, or merely a distance on $X$, if it verifies that $d(x, x)=0$ for all $x \in X$.

Diameters of subsets, coverings, etc. with respect to a distance function are defined in the same way that for a metric.

The definition that follows is going to be called as the second version of the fractal dimension definition.

Definition 4.2. Let $\boldsymbol{\Gamma}$ be a fractal structure on a distance space $(X, \rho)$ and let $N_{n}(F)$ be the number of elements of $\mathcal{A}_{n}(F)$ for all $n \in \mathbb{N}$. Thus, the (lower/upper) fractal dimensions II of a non-empty bounded subset $F$ of $X$ respectively, are defined as the (lower/upper) limit:

$$
\operatorname{dim}_{\Gamma}^{2}(F)=\lim _{n \rightarrow \infty} \frac{\log N_{n}(F)}{-\log \delta\left(F, \Gamma_{n}\right)}
$$

if this limit exists, where $\mathcal{A}_{n}(F)$ is defined at 3.2 for all $n \in \mathbb{N}$. 
It is also important that working with finite fractal structures simplifies the necessary calculations in order to determine the fractal dimension of a subset. In this way, the next remark shows that metrizable and separable topological spaces are useful for our purposes.

Remark 4.3. A metrizable space is second-countable if and only if it is separable (see [8, Theorem 5.7]). On the other hand, it is known that every topological space which is second-countable has a compatible finite fractal structure (see [7, Theorem 4.3]).

The second model we have presented in order to calculate the fractal dimension of a given subset enables the use of fractal structures whose elements could present different diameters on each level. In this way, we have used the quantity $\delta\left(F, \Gamma_{n}\right)$ in order to do this. Nevertheless, it would be also possible to consider $\delta\left(\Gamma_{n}\right)$ instead of $\delta\left(F, \Gamma_{n}\right)$ but it implies certain disadvantages. This idea is included in the next example.

Example 3. Let $\Gamma$ be a finite fractal structure whose levels are given by $\Gamma_{n}=\left\{\left[\frac{k_{1}}{2^{n}}, \frac{k_{1}+1}{2^{n}}\right] \times \ldots \times\left[\frac{k_{d}}{2^{n}}, \frac{k_{d}+1}{2^{n}}\right]: k_{i} \in\left\{-n 2^{n}, \ldots, n 2^{n}-1\right\}, i \in\right.$ $\{1, \ldots, d\}\} \cup\left\{\mathbb{R}^{d} \backslash(-n, n)^{d}\right\}$ for all natural number $n$, defined on the euclidean space $\mathbb{R}^{d}$. Thus, for all subset $F$ of $\mathbb{R}^{d}$, we have that $\delta\left(\Gamma_{n}\right)=$ $\infty$ for all $n \in \mathbb{N}$, but nevertheless, there exists a natural number $n_{0}$ such that $\delta\left(F, \Gamma_{n}\right)<\infty$ for all $n \geq n_{0}$.

Taking into account the definition 4.2 as well as the 3.2 one, it results clear that fractal dimensions I and II are going to agree if we select any fractal structure $\boldsymbol{\Gamma}$ such that $\delta\left(F, \Gamma_{n}\right)=\frac{1}{2^{n}}$ for all $n \in \mathbb{N}$. In this way, we are looking for a general condition for the elements of the fractal structure $\boldsymbol{\Gamma}$, in order to show that the two fractal dimension definitions are equal. First of all, we are going to pay attention to starbase fractal structures defined on a suitable class of distance spaces. In particular, a semimetric on a topological space $X$ (see [14, Def. 9.5]) is a nonnegative real mapping $d$ defined on $X \times X$, and verifying the following conditions: (i) $d(x, y)=0$ if and only if $x=y$, (ii) the mapping $d$ is symmetric, and (iii) The family $\left\{B_{d}(x, \varepsilon): \varepsilon>0\right\}$ is a neighborhood base for all $x \in X$, namely, the topology induced by the semimetric $d$ agrees with the starting topology. By the way, it is also possible to define a suitable semimetric on a GF-space. Note that starbase fractal structures enables to verify the condition (iii). The concept of semimetric associated with a fractal structure $\boldsymbol{\Gamma}$ was introduced on [9. Theorem 6.5]. Indeed, let $(X, \boldsymbol{\Gamma})$ be a starbase GF-space. The semimetric associated with the fractal structure $\Gamma$ can be described as 
the mapping $\rho: X \times X \rightarrow \mathbb{R}^{+}$given by:

$$
\rho(x, y)=\left\{\begin{array}{lll}
0 & \ldots & x=y \\
\frac{1}{2^{n}} & \ldots & y \in S t\left(x, \Gamma_{n}\right) \backslash S t\left(x, \Gamma_{n+1}\right) \\
1 & \ldots & y \notin S t\left(x, \Gamma_{1}\right)
\end{array}\right.
$$

It follows from expression 4.2 that $B_{\rho}\left(x, \frac{1}{2^{n}}\right)=S t\left(x, \Gamma_{n+1}\right)$ for all $n \in \mathbb{N}$ and all $x \in X$, and since $\Gamma$ is a starbase fractal structure, we conclude that the topology induced by the semimetric $\rho$ agrees with the topology induced by the fractal structure.

Theorem 4.4. Let $(X, \Gamma)$ be a starbase GF-space equipped with the semimetric associated with the fractal structure $\boldsymbol{\Gamma}$, and let $F$ be a subset of $X$. Suppose also that for all $n \in \mathbb{N}$ there exists $x \in F$ such that $S t\left(x, \Gamma_{n}\right) \neq S t\left(x, \Gamma_{n+1}\right)$. Then,

(1) $\overline{\operatorname{dim}}_{\Gamma}^{1}(F)=\overline{\operatorname{dim}}_{\Gamma}^{2}(F)$.

(2) $\underline{\operatorname{dim}}_{\Gamma}^{1}(F)=\underline{\operatorname{dim}}_{\Gamma}^{2}(F)$.

(3) If there exists any of the fractal dimensions I or II, then $\operatorname{dim}_{\Gamma}^{1}(F)=$ $\operatorname{dim}_{\Gamma}^{2}(F)$.

Proof. Given $n \in \mathbb{N}$, there exists $x \in F$ such that $S t\left(x, \Gamma_{n}\right) \neq S t\left(x, \Gamma_{n+1}\right)$. This implies that there exists $A \in \Gamma_{n}$ with $x \in A$ and such that $A \nsubseteq S t\left(x, \Gamma_{n+1}\right)$. Thus, $\delta(A)=\frac{1}{2^{n}}$, and then, $\delta\left(F, \Gamma_{n}\right)=\frac{1}{2^{n}}$. Therefore, $\varlimsup_{n \rightarrow \infty} \frac{\log N_{n}(F)}{-\log \delta\left(F, \Gamma_{n}\right)}=\varlimsup_{n \rightarrow \infty} \frac{\log N_{n}(F)}{n \log 2}$. The case for lower limits may be dealt with in the same way.

Fractal dimension II also generalizes the fractal dimension I as well as the box-counting dimension on the euclidean space $\mathbb{R}^{d}$ equipped with its natural fractal structure. In this way, we get a result like theorem 3.3 on the previous section.

Theorem 4.5. Let $\boldsymbol{\Gamma}$ be the natural fractal structure on the euclidean space $\mathbb{R}^{d}$, and let $F$ be a subset of $\mathbb{R}^{d}$. Then $\operatorname{dim}_{\Gamma}^{1}(F)=\operatorname{dim}_{\Gamma}^{2}(F)=$ $\operatorname{dim}_{B}(F)$ (the same is true for upper and lower dimensions).

Proof. By theorem 3.3, $\overline{\operatorname{dim}}_{\Gamma}^{1}(F)=\overline{\operatorname{dim}}_{B}(F)$.

Note also that $\delta(A)=\frac{\sqrt{d}}{2^{n}}$ for all $A \in \Gamma_{n}$. Accordingly, we have that $\varlimsup_{n \rightarrow \infty} \frac{\log N_{n}(F)}{-\log \delta\left(F, \Gamma_{n}\right)}=\varlimsup_{n \rightarrow \infty} \frac{\log N_{n}(F)}{n \log 2}$ which implies that $\overline{\operatorname{dim}}_{\Gamma}^{1}(F)=$ $\overline{\operatorname{dim}}_{\boldsymbol{\Gamma}}^{2}(F)$. The case for lower limits may be dealt with in the same way. 
As seen on proposition 3.4 for fractal dimension I, we can also study some analytical properties for the fractal dimension II definition. In this way, let $\boldsymbol{\Gamma}$ be a fractal structure on a distance space $(X, \rho)$. First of all, it is clear that both $\underline{\operatorname{dim}}_{\Gamma}^{2}$ and $\overline{\operatorname{dim}}_{\Gamma}^{2}$ are monotonic. Furthermore, since fractal dimension II generalizes fractal dimension I (by means of theorem 4.4), then any counterexample available for fractal dimension I remains valid for fractal dimension II. In particular, we can use those given for statements 3 , 4 and 5 on proposition 3.4 for fractal dimension II. Nevertheless, unlike fractal dimension I, we affirm that fractal dimension II does not verify the finite stability, as shown in the next example.

Example 4. Let $\Gamma_{1}$ be the natural fractal structure on $C_{1}$ as a selfsimilar set, where $C_{1}$ is the middle third Cantor set on $[0,1]$. Let also $\Gamma_{2}$ be a fractal structure on $C_{2}=[2,3]$ given by $\Gamma_{2}=\left\{\Gamma_{2, n}: n \in \mathbb{N}\right\}$, with $\Gamma_{2, n}=\left\{\left[\frac{k}{2^{2 n}}, \frac{k+1}{2^{2 n}}\right]: k \in\left\{2^{2 n+1}, 2^{2 n+1}+1, \ldots, 3 \cdot 2^{2 n}-1\right\}\right\}$ for all natural number $n$. Consider now $\Gamma=\left\{\Gamma_{n}: n \in \mathbb{N}\right\}$ as a fractal structure on $C=C_{1} \cup C_{2}$, where $\Gamma_{n}=\Gamma_{1, n} \cup \Gamma_{2, n}$ for all $n \in \mathbb{N}$. A simple calculation leads to $\operatorname{dim}_{\Gamma}^{2}\left(C_{1}\right)=\frac{\log 2}{\log 3}$ and $\operatorname{dim}_{\Gamma}^{2}\left(C_{2}\right)=1$, while $\operatorname{dim}_{\Gamma}^{2}(C)=\frac{\log 4}{\log 3}>1$.

Recall that on remark 3.9 we have shown that the fractal dimension I and the box-counting dimension of the middle third Cantor $C$ set $\operatorname{did}$ not agree: indeed, we got that $\operatorname{dim}_{B}(C)=\frac{\log 2}{\log 3}$, unlike $\operatorname{dim}_{\Gamma}^{1}(C)=1$. Note that these dimensions were calculated respect to different fractal structures: we used the natural fractal structure on the real line induced on $C$ in order to calculate the box-counting dimension, and on the other hand, we selected the natural fractal structure on the self-similar set to obtain the fractal dimension I of such space. However, the fractal dimension II agrees with the box-counting dimension of $C$. In this way, we consider again the natural fractal structure on $C$ as a self-similar set. Note that $\operatorname{dim}_{\Gamma}^{2}(C)=\lim _{n \rightarrow \infty} \frac{\log 2^{n}}{-\log 3^{-n}}=\frac{\log 2}{\log 3}=\operatorname{dim}_{B}(C)$ since on each level $\Gamma_{n}$ of the fractal structure there are $2^{n}$ elements with diameters equal to $3^{-n}$.

Nevertheless, though the value obtained from fractal dimension I of $C$ may seems counterintuitive at a first time, it is possible to explains it by means of the fractal dimension II. Indeed, the reason is that fractal dimension I only depends on the fractal structure we select in order to calculate it. We study this fact in the next remark.

Remark 4.6. Fractal dimension I only depends on the fractal structure, while fractal dimension II also depends on the diameter of the elements of each level of the fractal structure. We show this difference by constructing a family of spaces which from the fractal structure point of view are the same. 
Proof. Indeed, let us consider slight modifications on the construction of the middle third Cantor set, which we are going to denote by $C_{i}$, such that their associated contraction factors are $c_{i} \in\left[\frac{1}{3}, \frac{1}{2}\right)$ for the two similarities that give $C_{i}$. Thus, it is clear that $\delta\left(C_{i}, \Gamma_{n}\right)=c_{i}^{n}$ for all natural number $n$. Therefore, consider the natural fractal structure $\boldsymbol{\Gamma}_{i}$ on each space $C_{i}$ as a self-similar set. Then, an easy calculation yields (or apply theorem 4.15) $\operatorname{dim}_{B}\left(C_{i}\right)=\operatorname{dim}_{\Gamma}^{2}\left(C_{i}\right)=\frac{\log 2}{-\log c_{i}} \rightarrow 1=$ $\operatorname{dim}_{\Gamma}^{1}(C)$, when $c_{i} \rightarrow \frac{1}{2}$.

It is also possible to find an upper bound for the box-counting dimension of any subset in terms of its fractal dimension II, under the natural hypothesis $\delta\left(F, \Gamma_{n}\right) \rightarrow 0$. Furthermore, we find a connection between Hausdorff dimension and fractal dimension II.

Theorem 4.7. Let $\boldsymbol{\Gamma}$ be a fractal structure on a metric space $(X, \rho)$ with $F$ being a subset of $X$, and suppose that $\delta\left(F, \Gamma_{n}\right) \rightarrow 0$. Then,

(1) $\operatorname{dim}_{H}(F) \leq \underline{\operatorname{dim}}_{B}(F) \leq \underline{\operatorname{dim}}_{\Gamma}^{2}(F)$.

(2) If there exist both box-counting dimension and fractal dimension II of $F$, then $\operatorname{dim}_{B}(F) \leq \operatorname{dim}_{\Gamma}^{2}(F)$.

(3) Suppose also that there exists a constant c $>0$ such that $\delta\left(F, \Gamma_{n}\right) \leq$ c $\delta\left(F, \Gamma_{n+1}\right)$ for all natural number $n$. Then, $\overline{\operatorname{dim}}_{B}(F) \leq \overline{\operatorname{dim}}_{\Gamma}^{2}(F)$.

Proof. In order to calculate the box-counting dimension of $F$, let $N_{\delta}(F)$ be as (5) on equivalent box-counting definition seen at preliminary section 2 , and take $\delta_{n}=\delta\left(F, \Gamma_{n}\right)$ for all $n \in \mathbb{N}$.

(1) First of all, it is clear that $F \subset \cup\left\{A: A \in \mathcal{A}_{n}(F)\right\}$. Further, $\delta(A) \leq \delta\left(F, \Gamma_{n}\right)=\delta_{n}$ for all $A \in \mathcal{A}_{n}(F)$. Accordingly, $F$ can be covered by $N_{n}(F)$ sets with diameters at most $\delta_{n}$, so applying [[11],Prop. 4.1] we get: $\operatorname{dim}_{H}(F) \leq \underline{\operatorname{dim}}_{B}(F)=$ $\underline{\lim }_{n \rightarrow \infty} \frac{\log N_{n}(F)}{-\log \delta_{n}}=\underline{\operatorname{dim}}_{\Gamma}^{2}(F)$.

(2) It suffices with taking into account the previous result as well as the existence of both fractal dimensions.

(3) Let $c \in(0,1]$ be such that $\delta\left(F, \Gamma_{n}\right) \leq c \delta\left(F, \Gamma_{n+1}\right)$ for all $n \in \mathbb{N}$. Thus, $\delta\left(F, \Gamma_{n}\right)$ does not converges to 0 , which is a contradiction, so that $c>1$. Hence, there exists $d \in(0,1)$ such that $\delta_{n+1} \geq$ $d \delta_{n}$ for all $n \in \mathbb{N}$. Therefore, using remark $\left[2.6\right.$, $\overline{\operatorname{dim}}_{B}(F)=$ $\varlimsup_{n \rightarrow \infty} \frac{\log N_{\delta_{n}}(F)}{-\log \delta_{n}} \leq \varlimsup_{n \rightarrow \infty} \frac{\log N_{n}(F)}{-\log \delta\left(F, \Gamma_{n}\right)}$ since $\delta(A) \leq \delta_{n}$ for all $A \in \mathcal{A}_{n}(F)$. 
In this way, as a consequence of theorem 4.7, it is possible to get an approximation of the box-counting dimension of a self-similar set in terms of its fractal dimension II.

Corollary 4.8. Let $I=\{1, \ldots, m\}$ be a finite index set with $\left(\mathbb{R}^{d},\left\{f_{i}\right.\right.$ : $i \in I\})$ being an IFS whose associated self-similar set is $K$. Let also $\Gamma$ be the natural fractal structure on the self-similar set, and let $F$ be a subset of $K$. Then,

(1) $\underline{\operatorname{dim}}_{B}(F) \leq \underline{\operatorname{dim}}_{\Gamma}^{2}(F)$.

(2) If there exist both box-counting dimension and fractal dimension II of $F$, then $\operatorname{dim}_{B}(F) \leq \operatorname{dim}_{\Gamma}^{2}(F)$.

(3) Suppose that there exists $i \in I$ such that $f_{i}$ is a bilipschitz contractive mapping. Then $\overline{\operatorname{dim}}_{B}(F) \leq \overline{\operatorname{dim}}_{\Gamma}^{2}(F)$. In particular, this inequality is verified for strict self-similar sets.

Proof. In order to calculate the box-counting dimension of $F$, let $N_{\delta}(F)$ be as (5) on equivalent box-counting definition seen at preliminary section 2 .

(1) Since $K$ is a self-similar set, it is clear that $\delta\left(F, \Gamma_{n}\right) \rightarrow 0$ for all $F \subset K$, so it suffices with applying the theorem 4.7]1,

(2) This is by the first item.

(3) Let $f_{i}$ be a bilipschitz contraction. Thus, there exist constants $L_{i}$ and $c_{i}$ with $0<L_{i}<c_{i}<1$, such that $L_{i} d(x, y) \leq$ $d\left(f_{i}(x), f_{i}(y)\right) \leq c_{i} d(x, y)$ for all $x, y \in K$. Now, consider $A \in \mathcal{A}_{n}(F)$ verifying that $\delta(A)=\delta\left(F, \Gamma_{n}\right)$. By definition of supremum, we have that for all $\varepsilon>0$, there exist $x, y \in A$ such that $d(x, y)>\delta(A)-\varepsilon$. Let $B=f_{i}(A) \in \Gamma_{n+1}$. Thus, we have that $d\left(f_{i}(x), f_{i}(y)\right) \geq L_{i} d(x, y)>L_{i}(\delta(A)-\varepsilon)$, which implies that $\delta(B)=\delta\left(f_{i}(A)\right) \geq L_{i} \delta(A)$. Accordingly, $\delta\left(F, \Gamma_{n+1}\right) \geq \delta(B) \geq L_{i} \delta\left(F, \Gamma_{n}\right)$, so that it can be applied the proposition 4.7/3 in order to get the result.

In the next result, we look for properties on the natural fractal structure of an euclidean space in order to generalize theorem 4.5.

Theorem 4.9. Let $\boldsymbol{\Gamma}$ be a fractal structure on a metric space $(X, \rho)$ with $F$ being a subset of $X$, and suppose that there exists a natural number $k \in \mathbb{N}$ such that for all $n \in \mathbb{N}$, every subset $A$ of $X$ with $\delta(A) \leq \delta\left(F, \Gamma_{n}\right)$, meets at most at $k$ elements of the level $\Gamma_{n}$ on the fractal structure $\boldsymbol{\Gamma}$. Suppose also that $\delta\left(F, \Gamma_{n}\right) \rightarrow 0$. Then, 
(1) $\underline{\operatorname{dim}}_{B}(F) \leq \underline{\operatorname{dim}}_{\Gamma}^{2}(F) \leq \overline{\operatorname{dim}}_{\Gamma}^{2}(F) \leq \overline{\operatorname{dim}}_{B}(F)$. Moreover, if there exists $\operatorname{dim}_{B}(F)$, then $\operatorname{dim}_{B}(F)=\operatorname{dim}_{\Gamma}^{2}(F)$.

(2) If there exists $c \in(0,1)$ such that $\delta\left(F, \Gamma_{n+1}\right) \geq c \delta\left(F, \Gamma_{n}\right)$, then $\overline{\operatorname{dim}}_{B}(F)=\overline{\operatorname{dim}}_{\Gamma}^{2}(F)$ and $\underline{\operatorname{dim}}_{B}(F)=\underline{\operatorname{dim}}_{\Gamma}^{2}(F)$.

Proof. In order to calculate the box-counting dimension of $F$, let $N_{\delta}(F)$ be as (5) on equivalent box-counting definition seen at preliminary section 2 , and let $\delta_{n}=\delta\left(F, \Gamma_{n}\right)$ for all $n \in \mathbb{N}$.

(1) First of all, note that by proposition 4.7 we have that $\underline{\operatorname{dim}}_{B}(F) \leq$ $\underline{\operatorname{dim}}_{\Gamma}^{2}(F)$. On the other hand, the main hypothesis implies that $N_{n}(F) \leq k N_{\delta_{n}}(F)$ for all $n \in \mathbb{N}$, so it is clear that $\varlimsup_{n \rightarrow \infty} \frac{\log N_{n}(F)}{-\log \delta_{n}} \leq \varlimsup_{\lim _{n \rightarrow \infty}} \frac{\log N_{\delta_{n}}(F)}{-\log \delta_{n}} \leq \overline{\operatorname{dim}}_{B}(F)$.

(2) Note that, by remark 2.6, $\overline{\operatorname{dim}}_{B}(F)=\varlimsup_{n \rightarrow \infty} \frac{\log N_{\delta_{n}}(F)}{-\log \delta_{n}}$, which implies $\overline{\operatorname{dim}}_{B}(F) \leq \overline{\operatorname{dim}}_{\Gamma}^{2}(F)$. Now, an application of the first item leads to the opposite inequality. The case for lower limits may be dealt with in the same way.

The condition used on theorem 4.9 in order to get the equality between fractal dimension II and box-counting dimension is necessary, as the next remark shows:

Remark 4.10. There exists a self-similar set $K$ on the euclidean plane provided with its natural fractal structure $\boldsymbol{\Gamma}$, for which $\operatorname{dim}_{B}(K) \neq$ $\operatorname{dim}_{\Gamma}^{2}(K)$.

Proof. Indeed, let $I=\{1, \ldots, 8\}$ be a finite index set and let $\left(K,\left\{f_{i}\right.\right.$ : $i \in I\}$ ) be an IFS whose associated strict self-similar set is the unit square on the euclidean plane. Consider the contraction mappings $f_{i}: \mathbb{R}^{2} \rightarrow \mathbb{R}^{2}$ given as follows:

$$
f_{i}(x, y)=\left\{\begin{array}{lll}
\left(\frac{x}{2}, \frac{y}{4}\right)+\left(0, \frac{i-1}{4}\right) \ldots & i=1,2,3,4 \\
\left(\frac{x}{2}, \frac{y}{4}\right)+\left(\frac{1}{2}, \frac{i-5}{4}\right) \ldots & i=5,6,7,8
\end{array}\right.
$$

Let $\boldsymbol{\Gamma}$ be the natural fractal structure on $K$ as a self-similar set.

First of all, it is clear that $K$ is a self-similar set which is not a strict one. Further, we have that the contractive mappings $f_{i}$ have the same contraction factors, namely $c_{i}=\frac{1}{2}$ for all $i \in I$. It is also immediate the fact that $\operatorname{dim}_{B}(K)=2$. On the one hand, note that there are $8^{n}$ rectangles on each level $\Gamma_{n}$ of the fractal structure $\Gamma$, whose dimensions are $\frac{1}{2^{n}} \times \frac{1}{2^{2 n}}$. Thus, it is verified that $\delta(A)=\delta\left(K, \Gamma_{n}\right)=\sqrt{\frac{1+2^{2 n}}{2^{4 n}}}$ for all $A \in \Gamma_{n}$. Hence, we can calculate the fractal dimension II of $K$ as 
follows: $\lim _{n \rightarrow \infty} \frac{\log N_{n}(K)}{-\log \delta\left(K, \Gamma_{n}\right)}=\lim _{n \rightarrow \infty} \frac{3 n \log 2}{-\frac{1}{2} \log \left(\frac{1+2^{2 n}}{2^{4 n}}\right)}=\lim _{n \rightarrow \infty} \frac{3 n \log 2}{n \log 2}=$ 3.

Let $f, g: \mathbb{N} \rightarrow \mathbb{R}^{+}$be two sequences of positive real numbers. We say that $\mathcal{O}(f)=\mathcal{O}(g)$ iff they verify that $0<\lim _{n \rightarrow \infty} \frac{f(n)}{g(n)}<\infty$. In order to show that fractal dimensions I and II agree on a GF-space, we will need that all the elements on $\Gamma_{n}$ have a diameter of order $\frac{1}{2^{n}}$, as the next theorem establishes. Its proof is left to the reader.

Theorem 4.11. Let $\boldsymbol{\Gamma}$ be a fractal structure on a metric space $(X, \rho)$. Let $F$ be a subset of $X$, and suppose that $\delta(A)=\delta\left(F, \Gamma_{n}\right)$ for all $A \in$ $\mathcal{A}_{n}(F)$ and $\mathcal{O}\left(\delta\left(F, \Gamma_{n}\right)\right)=\mathcal{O}\left(\frac{1}{2^{n}}\right)$ for all $n \in \mathbb{N}$. Then $\operatorname{dim}_{\Gamma}^{1}(F)=$ $\operatorname{dim}_{\boldsymbol{\Gamma}}^{2}(F)$ (the same is true for upper and lower dimensions).

Recall that we have been able to calculate an upper bound for the box-counting dimension of every self-similar set equipped with its natural fractal structure in terms of its fractal dimension II. However, it is possible to reach the equality between these two quantities under certain conditions on the self-similar set structure. In particular, we will get the result if the elements on each level of $\boldsymbol{\Gamma}$ do not overlap too much, and because of the shape that the elements of the natural fractal structure on a self-similar set have, this restriction is going to be associated with the contractions $f_{i}$ of the corresponding IFS. Indeed, this property is the so called open set condition (see [11] and [23]):

Definition 4.12. Let $I=\{1, \ldots, m\}$ be a finite index set with $\left(X,\left\{f_{i}\right.\right.$ : $i \in I\})$ being an IFS whose associated self-similar set is $K$. The contractions $f_{i}$ satisfy the open set condition iff there exists a non-empty bounded open set $V$ of $X$ such that $\bigcup_{i \in I} f_{i}(V) \subset V$, with $f_{i}(V) \cap$ $f_{j}(V)=\emptyset$ for all $i \neq j$. Furthermore, if $V \cap K \neq \emptyset$, then the contractions $f_{i}$ are said to verify the strong open set condition.

Remark 4.13. Open set condition and strong open set condition are equivalent in any euclidean space (see [23]).

Lemma 4.14. Let $I=\{1, \ldots, m\}$ be a finite index set and let $\left(\mathbb{R}^{d},\left\{f_{i}\right.\right.$ : $i \in I\})$ be an IFS whose associated self-similar set is $K$. Suppose that $f_{i}$ are injective contractions verifying the open set condition for all $i \in I$. Then, there exists $\varepsilon>0$ and $x \in K$ such that for all natural number $n \in \mathbb{N}$ and all $\omega, u \in I^{n}$ with $\omega \neq u$, we have that $f_{\omega}(B(x, \varepsilon)) \cap f_{u}(B(x, \varepsilon))=\emptyset$, where $B(x, \varepsilon)$ denotes the ball centered at $x \in X$ with radius $\varepsilon>0$, which is embedded in $\mathbb{R}^{d}$.

Proof. Since $K \subset \mathbb{R}^{d}$ is a self-similar set, then we have that open set condition is equivalent to strong open set condition by remark 4.13 . Thus, there exists a non-empty bounded open set $O \subset \mathbb{R}^{d}$ such that 
$\cup_{i \in I} f_{i}(O) \subset O$, with $f_{i}(O) \cap f_{j}(O)=\emptyset$ for all $i, j \in I$ such that $i \neq j$. Moreover, $O \cap K \neq \emptyset$, so we can take $x \in O \cap K \subset O$. Since $O$ is an open set, there exists $\varepsilon>0$ such that $B=B(x, \varepsilon) \subset O$. We also know that $f_{i}(B) \subset O$ for all $i \in I$. The result will be shown by induction over the lenght of the words on $I^{n}$. In fact, let $\omega_{n}=i_{n} i_{n-1} \ldots i_{1} \in I^{n}$ and denote $f_{\omega_{n}}(F)=f_{i_{n}} \circ f_{i_{n-1}} \circ \ldots \circ f_{i_{1}}(F)$ for all $F \subset \mathbb{R}^{d}$. First of all, take $n=1$. Then we can select $\omega_{1}=i_{1}, u_{1}=j_{1} \in I$. Accordingly, we have that $f_{\omega_{1}}(B) \cap f_{u_{1}}(B) \subset f_{\omega_{1}}(O) \cap f_{u_{1}}(O)=\emptyset$, since the $f_{i}$ contractions verify the open set condition for all $i \in I$. Suppose now that $f_{\omega_{n}}(B) \cap f_{u_{n}}(B)=\emptyset$, with $\omega_{n}, u_{n} \in I^{n}$ such that $\omega_{n} \neq u_{n}$. Then, we distinguish the two following cases:

(1) Suppose that $i_{n+1}=j_{n+1}$, so that $\omega_{n+1}=i_{n+1} i_{n} \ldots i_{1}$ and $u_{n+1}=i_{n+1} j_{n} \ldots j_{1}$, with $\omega_{n+1}, u_{n+1} \in I^{n+1}$. Thus, using the injectivity of $f_{i_{n+1}}$ and the induction hypothesis, we conclude that $f_{\omega_{n+1}}(B) \cap f_{u_{n+1}}(B)=f_{i_{n+1}}\left(f_{\omega_{n}}(B)\right) \cap f_{i_{n+1}}\left(f_{u_{n}}(B)\right)=\emptyset$.

(2) Suppose that $\omega_{n+1} \neq u_{n+1}$, so that $\omega_{n+1}=i_{n+1} i_{n} \ldots i_{1}$ and $u_{n+1}=j_{n+1} j_{n} \ldots j_{1}$, with $i_{n+1} \neq j_{n+1}$. Then, it is clear the following chain of inclusions: $f_{w_{n+1}}(B) \cap f_{u_{n+1}}(B)=$ $f_{i_{n+1}}\left(f_{\omega_{n}}(B)\right) \cap f_{j_{n+1}}\left(f_{u_{n}}(B)\right) \subset f_{i_{n+1}}(O) \cap f_{j_{n+1}}(O)=\emptyset$. Note that $f_{\omega_{n}}(B) \subset O$ for all $\omega_{n} \in I^{n}$. Indeed, it is clear for words whose length is 1 , since it is obvious that $f_{i_{1}}(B) \subset O$. Suppose now that $f_{\omega_{n}}(B) \subset O$ for all $\omega_{n} \in I^{n}$. Then, it is verified that $f_{\omega_{n+1}}(B)=f_{i_{n+1}}\left(f_{\omega_{n}}(B)\right) \subset f_{i_{n+1}}(O) \subset O$.

On [11, Theorem 9.3] we find an interesting result which allows to calculate the box-counting dimension of a certain class of self-similar sets on the euclidean space $\mathbb{R}^{d}$ as the solution of a simple equation which only involves the contraction factors associated with each mapping $f_{i}$ of the corresponding IFS. Indeed, under the open set condition hypothesis, box-counting dimension agrees with Hausdorff dimension of such self-similar sets, and moreover, this value can be easily calculated from the mentioned expression. In this way, it would be an interesting result to reach the equality between box-counting dimension and fractal dimension II of a self-similar set whose contractions $f_{i}$ verify the open set condition. Moreover, the calculus of such quantity would become almost immediate from the number of contractive mappings of the IFS and its contraction factors.

Indeed, by means of the previous lemma, we present now the expected theorem.

Theorem 4.15. Let $I=\{1, \ldots, m\}$ be a finite index set and let $\left(\mathbb{R}^{d},\left\{f_{i}: i \in I\right\}\right)$ be an IFS whose associated strict self-similar set 
is $K$. Suppose that the similarities $f_{i}$ verify the open set condition and have equal similarity factors $c \in(0,1)$. Let $\boldsymbol{\Gamma}$ be the natural fractal structure on $K$ as a self-similar set. Then,

$$
\operatorname{dim}_{B}(K)=\operatorname{dim}_{\Gamma}^{2}(K)=\frac{-\log m}{\log c}
$$

Proof. In order to calculate the box-counting dimension of $K$, let $N_{\delta}(K)$ be as (6) on the equivalent box-counting definition seen at preliminary section 2 , and let $\delta_{n}=\delta\left(K, \Gamma_{n}\right)$ for all $n \in \mathbb{N}$. First of all, corollary 4.8,3 implies that $\overline{\operatorname{dim}}_{B}(K) \leq \overline{\operatorname{dim}}_{\Gamma}^{2}(K)$. Next, we are going to show the opposite inequality. Note that $\delta_{n}=c^{n} \delta(K)$ for all natural number $n$, since $K$ is a strict self-similar set. Applying lemma 4.14, there are so many disjoint balls with radius $\varepsilon_{n}=c^{n} \varepsilon$ with $\varepsilon>0$, and centered in $K$, as the number of elements of $I^{n}$. Now, since $N_{\varepsilon_{n}}(K)$ is the largest number of such balls, it is obvious that the number of elements of $I^{n}$ is at most $N_{\varepsilon_{n}}(K)$, namely, $N_{n}(K) \leq N_{\varepsilon_{n}}(K)$. On the other hand, there exists $k>0$ such that $\delta\left(K, \Gamma_{n}\right)=k \varepsilon_{n}$. Indeed, it suffices with taking $k=\frac{\delta(K)}{\varepsilon}$. Therefore, it results clear that $\varlimsup_{n \rightarrow \infty} \frac{\log N_{n}(K)}{-\log \delta\left(K, \Gamma_{n}\right)} \leq \varlimsup_{n \rightarrow \infty} \frac{\log N_{\varepsilon_{n}}(K)}{-\log k \varepsilon_{n}}=$ $\varlimsup_{n \rightarrow \infty} \frac{\log N_{\varepsilon_{n}}(K)}{-\log \varepsilon_{n}} \leq \overline{\operatorname{dim}}_{B}(K)$. Now, we get the next chain of inequalities: $\underline{\operatorname{dim}}_{B}(K) \leq \underline{\operatorname{dim}}_{\Gamma}^{2}(K) \leq \overline{\operatorname{dim}}_{\Gamma}^{2}(K) \leq \overline{\operatorname{dim}}_{B}(K)$, where the first inequality is by corollary 4.8. Now, the existence of the box-counting dimension of $K$ implies the existence of the fractal dimension II of $K$ and the expected equality $\operatorname{dim}_{B}(K)=\operatorname{dim}_{\Gamma}^{2}(K)$. Furthermore, apply [11, Theorem 9.3] in order to get the last equality on 4.4.

The hypothesis based on the equality of the contraction factors on theorem 4.15] is necessary. By the example in remark 4.10, the contractions have to be similarities, while by the next example, all contraction factors must be the same.

Remark 4.16. There exists a strict self-similar set $K$, whose similarities $f_{i}$ verify the open set condition and have different contraction factors $c_{i}$, such that $\operatorname{dim}_{B}(K)<\operatorname{dim}_{\Gamma}^{2}(K)$.

Proof. Indeed, we can take the IFS ( $\left.\mathbb{R},\left\{f_{i}: i \in\{1,2\}\right\}\right)$, whose associated self-similar set is $K$, where the similarities $f_{1}, f_{2}: \mathbb{R} \rightarrow \mathbb{R}$ are given by $f_{1}: x \mapsto \frac{x}{2}$, and $f_{2}: x \mapsto \frac{x+3}{4}$. It is clear that their associated contraction factors are $c_{1}=\frac{1}{2}$ and $c_{2}=\frac{1}{4}$ respectively, and it is also obvious the fact that $K$ is a strict self-similar set. We can also justify that the similarities $f_{i}$ satisfy the open set condition on an easy way: it suffices with taking $V=(0,1)$ as a suitable open set. Now, let $\boldsymbol{\Gamma}$ be the natural fractal structure on $K$ as a self-similar set. Then, 
by [11, Theorem 9.3], we can calculate the box-counting dimension of $K$ as the solution of the equation $\frac{1}{2^{s}}+\frac{1}{4^{s}}=1$. Thus, we have that $\operatorname{dim}_{B}(K)=\frac{\log \left(\frac{1+\sqrt{5}}{2}\right)}{\log 2}$. On the other hand, it is clear that there are $2^{n}$ subintervals of $[0,1]$ on each level $\Gamma_{n}$ of the fractal structure $\boldsymbol{\Gamma}$, where the largest of them has diameter equal to $\frac{1}{2^{n}}$ for all natural number $n \in \mathbb{N}$, which implies that $\operatorname{dim}_{\boldsymbol{\Gamma}}^{2}(K)=1>\operatorname{dim}_{B}(K)$.

\section{An APPliCATiOn TO THE DOMAin OF WORDS}

The goal of this section consists of providing a variety of applications of the fractal dimension introduced in this paper, where the boxcounting dimension cannot be computed. Indeed, we show that the use of fractal structures in the context of a domain of words could contribute some information about the complexity of a language. In this way, we study and show how to calculate the fractal dimension of a language generated by means of a regular expression, and then we introduce an empirical application consisting of computing the fractal dimension of a natural language. In both cases, we show and explain the obtained results. Finally, we present how fractal structures and dimensions can help us in order to determine the efficiency of a system of information encoding like the BCD. We start with some preliminary topics.

5.1. Fractal structures and domains of words. The domain of words, which we introduce next, appears when modeling the streams of information in Kahn's model of parallel computation (see [17], 20]). Indeed, let $\Sigma$ be a finite non-empty alphabet (set) and let $\Sigma^{\infty}$ be the collection of finite $\left(\cup_{n \in \mathbb{N}} \Sigma^{n}\right)$ and infinite $\left(\Sigma^{\mathbb{N}}\right)$ sequences (called words) over $\Sigma$. Let us denote by $\varepsilon$ to the empty word.

The prefix order $\sqsubseteq$ is defined on $\Sigma^{\infty}$, as usual, by $x \sqsubseteq y$ iff $x$ is a prefix of $y$. Thus, for each $x \in \Sigma^{\infty}$, let $l(x)$ be the length of $x$, where $l(\varepsilon)=0$, and for $x, y \in \Sigma^{\infty}$, we denote by $x \sqcap y$ to the common prefix of $x$ and $y$. Hence, a (non-archimedean) quasi-metric $d$ can be defined on $\Sigma^{\infty}$ by $d(x, y)=0$ if $x \sqsubseteq y$, and $d(x, y)=2^{-l(x \sqcap y)}$, in other case (see [26]). Furthermore, the non-archimedean quasi-metric $d$ induces a fractal structure on $\Sigma^{\infty}$ (see [5]) which can be described as follows: $\boldsymbol{\Gamma}=\left\{\Gamma_{n}\right.$ : $n \in \mathbb{N}\}$, where its levels are given by

$$
\Gamma_{n}=\left\{w^{\#}: w \in \Sigma^{n}\right\} \cup\left\{w^{\sqsubseteq}: w \in \Sigma^{k}: k<n\right\}
$$

Note that for $w \in \Sigma^{n}, w \sqsubseteq=\left\{u \in \Sigma^{k}: k \leq n\right.$ and $\left.u \sqsubseteq w\right\}$ is the collection of prefixes of $w$, and $w^{\#}=\left\{w u: u \in \Sigma^{\infty}\right\} \cup w^{\sqsubseteq}$ is the collection of words (finite or infinite) which start with $w$ or are a prefix of $w$. Moreover, for each $w \in \Sigma^{n}$, we have that $w^{\#}=B_{d^{-1}}\left(w, 2^{-n}\right)$, and for each $w \in \Sigma^{k}$ with $k<n, w^{\sqsubseteq}=B_{d^{-1}}\left(w, 2^{-n}\right)$. 
Then, a language $L$ is defined as a subspace of $\Sigma$, and usually it is defined by means of a formal grammar. In particular, we can consider the languages generated by regular expressions. Now, since we have a fractal structure and a quasi-metric, then we can calculate the fractal dimension of any language.

5.2. The fractal dimension of a language generated by a regular expression. Consider the regular expression $(00+1)^{+}$, that is constructed by concatenating consecutively (at least one time) 00 and 1. Our main purpose consists of computing the fractal dimension of the previous language $L \subset \Sigma^{\infty}$ generated by means of the previous regular expression. In this way, we are going to apply the fractal dimension I model. First of all, note that $L$ can be described as the following set:

$$
L=\{1,00,11,100,001,111,0000,0011,1001,1100,1111, \ldots\}
$$

Let also $\Gamma$ be the fractal structure induced by the non-archimedean quasi-metric $d$ given at 5.1. Then, we have that $\Gamma=\left\{\Gamma_{n}: n \in \mathbb{N}\right\}$, where, for instance, the first levels are given as follows: $\Gamma_{1}=\left\{1^{\#}, 0^{\#}\right\}$, $\Gamma_{2}=\left\{10^{\#}, 11^{\#}, 00^{\#}, 1 \sqsubseteq\right\}, \Gamma_{3}=\left\{000^{\#}, 001^{\#}, 100^{\#}, 111^{\#}, 110^{\#}, 00^{\sqsubseteq}\right.$, $\left.11^{\sqsubseteq}, 1^{\sqsubseteq}\right\}$, and so on. In order to calculate the fractal dimension of $L$, note that $N_{1}(L)=2, N_{2}(L)=3+1, N_{3}(L)=5+3, N_{4}(L)=8+6$, $N_{5}(L)=13+11$, and so on, where the first term on each sum refers to the number of elements of the appropiate level of the form $w^{\#}$, and the second one to the number of elements of each level of the form $w^{\sqsubseteq}$. Hence, if we consider $\left\{f_{n}\right\}_{n \in \mathbb{N}}$ as the Fibonacci's sequence, where $f_{1}=f_{2}=1$, with $f_{n}=f_{n-1}+f_{n-2}$ for all $n \geq 3$, then it can be checked what follows:

$$
N_{n}(L)=f_{n+2}+\sum_{i=2}^{n} f_{i}
$$

for all $n \in \mathbb{N}$. In this way, since the Fibonacci's sequence verifies the next property:

$$
\sum_{i=1}^{n} f_{i}=f_{n+2}-1 \text { for all } n \geq 2
$$

then $N_{n}(L)=2\left(f_{n+2}-1\right)$ for all natural number $n$. Furthermore, it is known that $f_{n}=\frac{\varphi^{n}-\beta^{n}}{\varphi-\beta}$ for all $n \in \mathbb{N}$, where $\varphi=\frac{1+\sqrt{5}}{2}$ (the golden ratio) and $\beta=\frac{1-\sqrt{5}}{2}$. Accordingly, we obtain the fractal dimension I of $L$ as follows:

$$
\begin{aligned}
\lim _{n \rightarrow \infty} \frac{\log N_{n}(L)}{n \log 2} & =\lim _{n \rightarrow \infty} \frac{\log f_{n+2}}{n \log 2}=\lim _{n \rightarrow \infty} \frac{\log \left(\varphi^{n+2}-\beta^{n+2}\right)}{n \log 2}= \\
& =\lim _{n \rightarrow \infty} \frac{\log \left(1-\left(\frac{\beta}{\varphi}\right)^{n+2}\right)+(n+2) \log \varphi}{n \log 2}=\log _{2} \varphi
\end{aligned}
$$


Hence, we have that the fractal dimension of $L$ is related with the golden ratio. Note that the result we have just got in expression 5.4 implies that $N_{n}(L) \simeq \varphi^{n}$ for all natural number $n$, which is equivalent to $N_{n+1}(L) \simeq \varphi \cdot N_{n}(L)$. Roughly speaking, we have that for any word of length $n$, there are about $\varphi$ words of length $n+1$. On the other hand, since $\operatorname{dim}_{\Gamma}^{1}\left(\Sigma^{\infty}\right)=1$, it is clear that $N_{n+1}\left(\Sigma^{\infty}\right) \simeq 2 \cdot N_{n}\left(\Sigma^{\infty}\right)$ for all $n \in \mathbb{N}$. Thus, fractal dimension I constitutes a register about the evolution and complexity of the language $L$ with respect to the domain of words $\Sigma^{\infty}$ where it has been defined.

Moreover, since $\delta\left(L, \Gamma_{n}\right)=\frac{1}{2}$ for all $n \in \mathbb{N}$, then we have that $\operatorname{dim}_{\Gamma}^{2}(L)=\infty$, so that the fractal dimension II method does not provide information about the evolution of the language $L$ with respect to the domain of words.

Fractal dimension II will be useful in order to describe the complexity of a language when the related fractal structure $\boldsymbol{\Gamma}$ is starbase. In this way, note that the fractal structure induced by the (non-archimedean) quasi-metric $d$ is not starbase. Indeed, suppose the opposite. Then, $\Sigma^{\infty}$ would be a metrizable space (see [7]), which implies that $\Sigma^{\infty}$ is $T_{1}$, that constitutes a contradiction. Now, by means of proposition 3.6, if the distance function is compatible with the fractal structure, we have that $\delta\left(\Gamma_{n}\right) \nrightarrow 0$ as $n \rightarrow \infty$. Further, if $N_{n}(L) \rightarrow \infty$ as $n \rightarrow \infty$ (as it occurs in this example), we get that $\operatorname{dim}_{\Gamma}^{2}(L)=\infty$. However, this disadvantage can be improved by means of the next remark.

Remark 5.1. Let $\boldsymbol{\Gamma}^{\prime}=\left\{\Gamma_{n}^{\prime}: n \in \mathbb{N}\right\}$ be the fractal structure whose levels are given by $\Gamma_{n}^{\prime}=\left\{w^{\#}: w \in \Sigma^{n}\right\}$ for all natural number $n$. Note that this fractal structure constitutes a simplification of the previous $\boldsymbol{\Gamma}$. Furthermore, we have that $N_{n}^{\prime}(L)=f_{n+2}$, as well as $\delta\left(L, \Gamma_{n}\right)=\frac{1}{2^{n}}$ for all $n \in \mathbb{N}$, which implies that $\operatorname{dim}_{\Gamma^{\prime}}^{2}(L)=\log _{2} \varphi=\operatorname{dim}_{\Gamma}^{1}(L)$, so that fractal dimension II provides the same information about $L$ than fractal dimension I in this case.

5.3. An empirical application to natural languages. Although fractal dimensions can be computed for languages described by means of regular expressions, where words of infinite length could exist, it is also possible to determine the fractal dimension of other languages, like the natural ones. In this way, since fractal dimensions are not only theoretical but also empirical quantities, we are going to calculate them with respect to the fractal structure $\boldsymbol{\Gamma}$ given at 5.1, though it is also possible to work with the fractal structure $\boldsymbol{\Gamma}^{\prime}$. Nevertheless, since always exists a maximum value for the length of any word in a natural language, it is clear that $\operatorname{dim}_{\boldsymbol{\Gamma}}^{1}(N)=\operatorname{dim}_{\boldsymbol{\Gamma}^{\prime}}^{1}(N)=0$ where $N$ denotes to any natural language. 
Taking it into account, for a practical application, we are going to calculate the fractal dimension of a given natural language $N$ by means of the slope of the regression line obtained by comparing $\log N_{n}(N)$ versus $\log 2^{n}$. In order to do this, we are going to count the number of words with a given length which appears on the relative dictionary to the natural language $N$. Note that in this case we are computing $N_{n}(N)$ for $n \in\{1,2,3,4\}$, which leads to a suitable approximation of the fractal dimension of $N$. This quantity provides information about the complexity and evolution of that natural language, by means of the length and the variety its words present (in this case, specially the words with small length). Indeed, we have calculated the fractal dimension of a wide list of natural languages and got the results which appear in the table 1.

\begin{tabular}{||l|c||}
\hline \hline Natural Language & Fractal dimension \\
\hline German, Polish & 3.1 \\
\hline U.K. English & 3.0 \\
\hline U.S. English, Hungarian, Italian, Latin & 2.9 \\
\hline Africans, Czech, Danish, Dutch & 2.8 \\
\hline Spanish, Swedish, Ukrainian & 2.7 \\
\hline Croatian, Mongolian, Portuguese, & \\
Romanian, Russian, Slovenian & 2.6 \\
\hline French, Serbian, Slovak & 2.5 \\
\hline Bulgarian & 2.4 \\
\hline \hline
\end{tabular}

TABLE 1. Fractal dimensions for natural languages, calculated respect to the fractal structure $\boldsymbol{\Gamma}$ induced by the quasi-metric $d$ (see 5.1).

The obtained results allows to compare the complexity between two given natural languages. For instance, in figure 1 we can regard the difference of the fractal complexity between English and Spanish. In this way, note that English has a larger variety of small prefixes than Spanish, although the number of prefixes of a given length increases faster in Spanish than in English for medium length words.

The fractal dimension models developed in this paper allows also to compare the complexity of any text written in a specific language. Indeed, since the fractal dimension of a language has been calculated by means of its corresponding dictionary, an effective calculation of the fractal dimension of a book written on that language gives us some interesting information about the variety of the words it uses. Moreover, it is also possible to perform a fractal complexity comparison between a given translation of a book and its original version. 


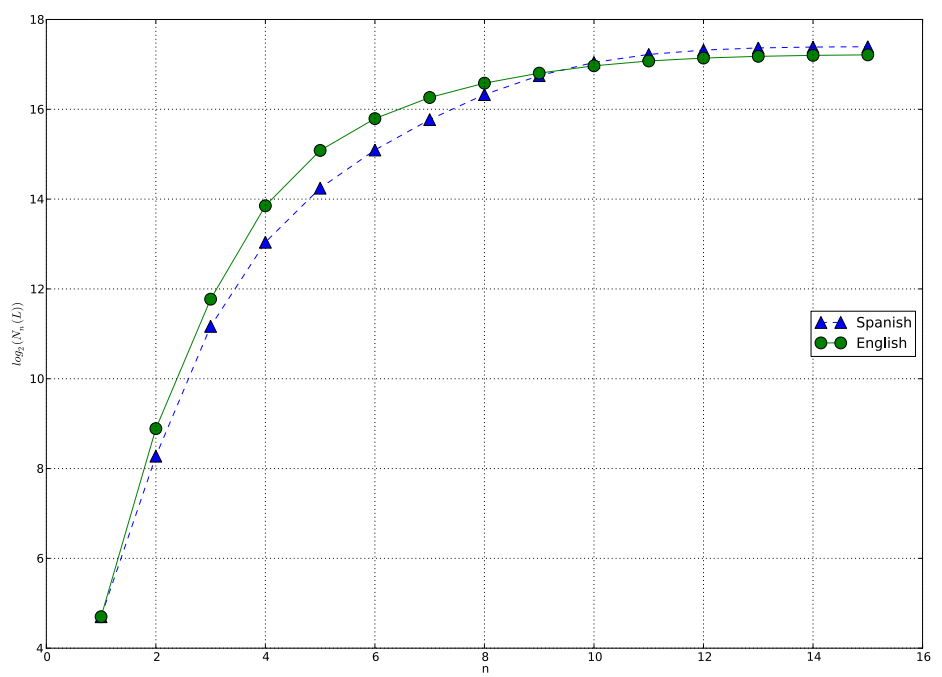

FiguRE 1. Graph of the fractal complexity comparison between the languages English and Spanish.

With this in mind, next we show some examples where the fractal dimension of some books has been calculated. In order to do this, we have considered the set which contains all the words used in the corresponding book and chosen the fractal structure $\boldsymbol{\Gamma}$ defined in 5.1 . The effective calculation of the fractal dimension has been done by taking into account the four first levels of the fractal structure. Thus, we show the fractal dimension of the selected books as well as the fractal dimension of that book's language (the latter is the number which appears into brackets), which gives information about the complexity of the given translations.

For instance, the fractal dimension of the English version of the book On the Origin of Species (Charles Darwin, 1859) is 2.2 (3.0), while the fractal dimension of its French version is about 2.3 (2.6). On the other hand, the fractal dimension of the English version of the text Alice's Adventures in Wonderland (Lewis Carroll, 1865) is 1.8 (3.0), while its Spanish version has the value 2.0 (2.7). Note that a classical book like Don Quijote de la Mancha (Miguel de Cervantes, 1605) has a fractal dimension of 2.5 (2.7) in its original Spanish version, while its English translation is about 2.3 (3.0), and for instance, the dimension of its Dutch version is $2.2(2.9)$.

5.4. The fractal dimension as a tool to study the efficiency of an encoding system. So far, we have shown two applications of our models for determining the fractal dimension where box-counting 
dimension has no sense. The first one results interesting since it consists on a language generated by means of a regular expression where infinite length words could exist. The second one consists of an empirical application where we use the fractal dimension as a tool in order to study the complexity and evolution of a natural language. Now, we are going to show a computational application of our models to an interesting method for coding and decoding information from other languages.

The binary-coded decimal (which we denote by BCD for short) is a method for encoding decimal numbers which represents each decimal digit by means of its binary sequence. Although nowadays is used with less frequency in some applications, it results useful in computer and electronic systems (which specially consists only of digital logic without microprocessors) in order to display or print decimal numbers. One of its advantages is that BCD allows faster decimal calculations. Nevertheless, it is not an efficient encoding method, since it uses more space than a simple binary representation.

The BCD system stores each decimal digit from 0 to 9 by means of 4 bits which contains its binary sequence. In this way, the set

$$
S=\{0000,0001,0010,0011,0100,0101,0110,0111,1000,1001\}
$$

generates the language $B$ of binary-coded decimal numbers. For instance, note that $1100 \notin B$, since it is not a codification of any decimal digit. For example, the number 215 (in 10-base), is converted to an expression like 001000010101 . Indeed, note that its binary representation is 11010111, which needs less space to be stored than by means of the BCD codification.

Fractal dimension constitutes a measure of the computational efficiency of a language which consists of the encoding of another language, like the BCD system. In this way, next we extract some conclusions related to the encoding goodness of the latter method. Let $\Gamma$ be the fractal structure defined at 5.1, though it is also possible to consider its simplified version $\boldsymbol{\Gamma}^{\prime}$. First of all, note that

$$
N_{n}(L)=\left\{\begin{array}{lll}
2 \cdot 10^{i} & \ldots & n=4 i \\
3 \cdot 10^{i} & \ldots & n=4 i+1 \\
4 \cdot 10^{i} & \ldots & n=4 i+2 \\
6 \cdot 10^{i} & \ldots & n=4 i+3
\end{array}\right.
$$

which leads to $\operatorname{dim}_{\boldsymbol{\Gamma}}^{1}(L)=\operatorname{dim}_{\boldsymbol{\Gamma}^{\prime}}^{1}(L)=\frac{\log 10}{\log 16}=\log _{2} \sqrt[4]{10}$. Therefore, we have that $N_{n}(L) \simeq 10^{\frac{n}{4}}$ for all $n \in \mathbb{N}$, which implies that $N_{n+1}(L) \simeq$ $\sqrt[4]{10} \cdot N_{n}(L)$ for all natural number $n$. Thus, given a real number with $n$ decimal digits, we need $4 n$ binary digits in order to encode it, which allows to represent $10^{n}$ different numbers on the BCD system, 
while in the binary representation, $4 n$ binary digits allows to represent $2^{4 n}$ numbers. Therefore, we can calculate the efficiency of BCD when encoding decimal numbers, by means of the next ratio:

$$
\frac{N_{n}(L)}{N_{n}\left(\Sigma^{\infty}\right)}=\frac{10^{\frac{n}{4}}}{2^{n}}=\left(\frac{10}{16}\right)^{\frac{n}{4}}
$$

For instance, take a decimal number with $n=10$ digits, which needs $4 n=40$ bits to be encoded. Accordingly,

$$
\frac{N_{40}(L)}{N_{40}\left(\Sigma^{\infty}\right)}=\left(\frac{10}{16}\right)^{10} \simeq \frac{10^{10}}{10^{12}}=0.01
$$

so that, $\mathrm{BCD}$ encodes the $1 \%$ of the possible numbers encoded directly in binary. Indeed, there is a lack of the order of $62.5 \%$ (that is, $\left(\frac{10}{16}\right)^{\frac{1}{4}}$ ) for each encoded digit.

\section{REFERENCES}

[1] J.W. Baish and R.K. Jain, Fractals and Cancer, Cancer Research 60 (2000), pp. 3683-3688.

[2] C. Bandt and T. Retta, Topological spaces admitting a unique fractal structure, Fund. Math. 141 (1992), 257-268.

[3] C.L. Alados, Y. Pueyo, M.L. Giner, T. Navarro, J. Escos, F. Barroso, B. Cabezudo and J.M. Emlen, Quantitative characterization of the regressive ecological succession by fractal analysis of plant spatial patterns, Ecological Modelling 163 (2003), pp. 1-17.

[4] E. de Amo, I. Chitescu, M. Díaz Carrillo and N. A. Secelean, A new approximation procedure for fractals, Journal of Computational and Applied Mathematics, Volume 151, Issue 2, 15 February 2003, pp. 355-370.

[5] F.G. Arenas and M.A. Sánchez-Granero, A characterization of nonarchimedeanly quasimetrizable spaces, Rend. Istit. Mat. Univ. Trieste, Suppl. Vol. XXX (1999), pp. 21-30.

[6] F.G. Arenas and M.A. Sánchez-Granero, A new approach to metrization, Topology Appl. 123 (2002), no. 1, pp. 15-26.

[7] F.G. Arenas and M.A. Sánchez-Granero, A new metrization theorem, Bollettino U.M.I (8) 5-B (2002), 109-122.

[8] F.G. Arenas and M.A. Sánchez-Granero, Completeness in metric spaces, Indian J. Pure Appl. Math. 33 (2002), no. 8, pp. 1197-1208.

[9] F.G. Arenas and M.A. Sánchez-Granero, A characterization of self-similar symbolic spaces, preprint.

[10] I. Chitescu, H. Georgescu and R. Miculescu, Approximation of infinite dimensional fractals generated by integral equations, Journal of Computational and Applied Mathematics, Volume 234, Issue 5, 1 July 2010, pp. 1417-1425.

[11] K. Falconer, Fractal Geometry, Mathematical Foundations and Applications, John Wiley \& Sons, 1990.

[12] X. Fan, H. Yang, Exponential attractor and its fractal dimension for a second order lattice dynamical system, Journal of Mathematical Analysis and Applications, Elsevier, Volume 367, Number 2, July 2008, pp. 350-359.

[13] P. Fletcher and W.F. Lindgren, Quasi-Uniform Spaces, Lecture Notes Pure Appl. Math. 77, Marcel Dekker, New York, 1982. 
[14] G. Gruenhage, Generalized metric spaces, chapter 10 of [K.Kunen, J.E. Vaughan (ed.), Handbook of set-theoretic topology, Elsevier Science Publishers B.V., 1984.].

[15] T. Hirata, A Correlation Between the $\mathrm{b}$ Value and the Fractal Dimension of Earthquakes, Journal of Geophysical Research, Vol. 94, No. B6, 1989, pp. 75077514.

[16] J. Hutchinson, Fractals and self-similarity, Indiana Univ. J. Math. 30 (1981), pp. 713-747.

[17] G. Kahn, The semantic of a simple language for parallel procesing, Proc. IFIP Congress 74, Elsevier, Amsterdam, 1974, 471-475.

[18] G. Landini, G.P. Misson and P.I. Murray, Fractal analysis of the normal human retinal fluorescein angiogram, Current Eye Research, Vol. 12, No. 1 (1993), pp. 23-27.

[19] K.-H. Lin, K.-M. Lam and W.-C. Siu, Locating the eye in human face images using fractal dimensions, IEE Proc.- Vis. Image Signal Process., Vol. 148, No. 6 (2001).

[20] S.G. Matthews, Partial metric topology, Proc. 8th Summer Conference on General Topology and Applications, Ann. New York Acad. Sci., vol. 728, New York Academy of Sciences, New York, 1994, 183-197.

[21] U.E. Ruttimann, R.L. Webber and J.B. Hazelrig, Fractal dimension from radiographs of peridental alveolar bone: a possible diagnostic indicator of osteoporosis, Oral surgery, oral medicine, oral pathology (1992), vol. 74 (1), pp. 98-110.

[22] M.A. Sánchez-Granero, Applications of fractal structures, Proceedings of the Workshop in Applied Topology WiAT'10, pp. 1-10.

[23] A. Schief, Separation properties for self-similar sets, Proc. Amer. Math. Soc. 122 (1994), Number 1, pp. 111-115.

[24] L. Sella and P. Collins, Computation of symbolic dynamics for one-dimensional maps, Journal of Computational and Applied Mathematics, Volume 234, Issue 2, 15 May 2010, pp. 418-436.

[25] S.D. Shore and S. Romaguera, Metrizability of asymmetric spaces, Ann. New York Acad. Sci. 806 (1996), pp. 382-392.

[26] M.B. Smyth, Quasi-uniformities: Reconciling domains with metric spaces,, in: M. Main, et al. (Eds.), Mathematical Foundations of Programming Language Semantics, 3rd Workshop, Tulane, 1987, in: Lecture Notes Computer Science, vol. 298, Springer, Berlin, 1988, 236-253.

Current address: Area of Geometry and Topology, Faculty of Science, Universidad de Almería, 04071 Almería, Spain

E-mail address: misanche@ual.es and fmm124@ual.es 